\title{
Probing the merger history of red early-type galaxies with their faint stellar substructures
}

\author{
Brisa Mancillas ${ }^{1}$, Pierre-Alain Duc ${ }^{2}$, Françoise Combes ${ }^{1,3}$, Frédéric Bournaud ${ }^{4}$, Eric Emsellem ${ }^{5,6}$, \\ Marie Martig ${ }^{7}$, and Leo Michel-Dansac ${ }^{6}$

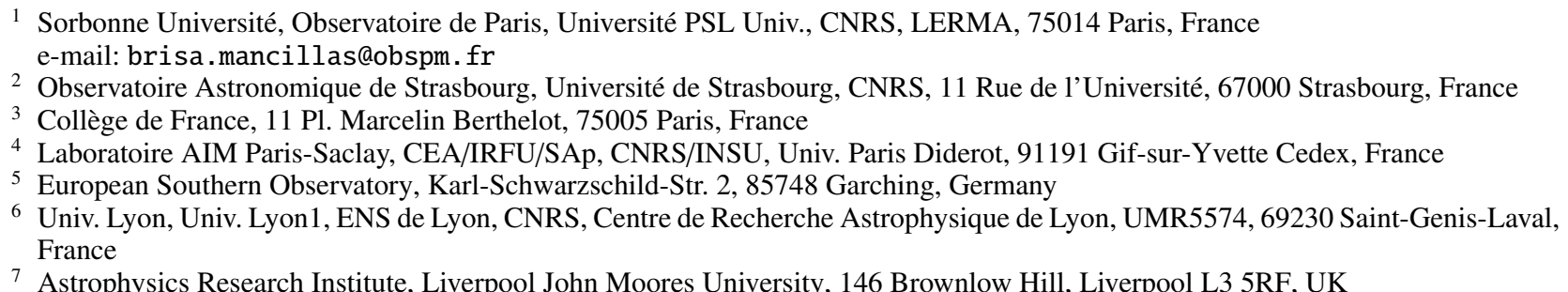 \\ 7 Astrophysics Research Institute, Liverpool John Moores University, 146 Brownlow Hill, Liverpool L3 5RF, UK
}

Received 15 July 2019 / Accepted 8 September 2019

\begin{abstract}
Several detailed observations, such as those carried out at the Canada-France-Hawaii Telescope (CFHT), have revealed prominent Low Surface Brightness (LSB) fine structures that lead to a change in the apparent morphology of galaxies. Previous photometry surveys have developed observational techniques which make use of the diffuse light detected in the external regions of galaxies. In these studies, the outer perturbations have been identified and classified. These include tidal tails, stellar streams, and shells. These structures serve as tracers for interacting events and merging events and retain some memory of the mass assembly of galaxies. Cosmological numerical simulations are required to estimate their visibility timescale, among other properties, in order to reconstruct the merger history of galaxies. In the present work, we analyze a hydrodynamical cosmological simulation to build up a comprehensive interpretation of the properties of fine structures. We present a census of several types of LSB fine structures compiled using a visual inspection of individual snapshots at various points in time. We reconstruct the evolution of the number of fine structures detected around an early-type galaxy and we compare it with the merger history of the galaxy. We find that most fine structures are associated with major and intermediate mass merger events. Their survival timescale ranges between 0.7 and 4 Gyr. Shells and streams remain visible for a longer time, while tidal tails have a shorter lifetime. These estimates for the survival time of collisional debris provide clues for the interpretation of the shape and frequency of fine structures observed in deep images with regard to their mass assembly. We find that the detectability of stellar streams is most sensitive at the surface brightness limit, demonstrating greater visibility at the deepest surface brightness level used in our simulation. We see between two and three times more streams based on a surface brightness cut of $33 \mathrm{mag} \mathrm{arcsec}-2$ than with $29 \mathrm{mag} \mathrm{arcsec}^{-2}$. We find that the detection of shells is strongly dependent upon the projection angle.
\end{abstract}

Key words. galaxies: elliptical and lenticular, cD - galaxies: evolution - galaxies: interactions - galaxies: peculiar galaxies: structure

\section{Introduction}

Within the frame of the Lambda cold dark matter $(\Lambda \mathrm{CDM})$ cosmological model, galaxy interactions play a major role in hierarchical galaxy formation theory. The models suggest that galaxies are assembled through successive mergers and continuous processes of cold gas and dark matter accretion (White \& Rees 1978; Bullock \& Johnston 2005; Naab et al. 2007; Cooper et al. 2013, 2015; Rodriguez-Gomez et al. 2016). Early studies propose that massive early-type galaxies result from a violent relaxation following the merger of two equal mass disk galaxies (Toomre 1977). Numerical studies demonstrate that, at high redshifts, the so-called intermediate-mass mergers of stellar massratio 4:1-10:1 could be progenitors of S0-like galaxies. In addition, multiple sequential mergers of mass ratios ranging from 4:1-50:1 or even 100:1 can lead to the build-up of elliptical galaxies (Bournaud et al. 2005, 2007; Stewart et al. 2008; Karademir et al. 2019). The main mode of mass growth for early-type galaxies is also dependent on redshift. It is expected that minor mergers, along with stellar accretion, dominate a galaxy's mass growth, rather than major mergers at $z<1$, since they occur more frequently (Kaviraj et al. 2009; Peirani et al. 2010; Hilz et al. 2012). Nonetheless, gas-rich mergers dominate the galaxy mass assembly in the early formation phases $(2<z<8)$ (e.g. Naab et al. 2007), although direct gas accretion from cold streams, as inferred from numerical simulations and, very likely, from observations (e.g. Argudo-Fernandez et al. 2016), may also play a key role in the building of galaxy disks.

Interactions have left vestiges of their existence in the outer regions of central galaxies from disrupted satellites and from equal-mass mergers (Helmi \& White 1999; Ferguson et al. 2002; Majewski et al. 2003). This collisional debris is mainly made up of stellar streams, plumes, tidal tails and stellar shells (Mihos et al. 2005; McConnachie et al. 2009; Janowiecki et al. 2010; Martínez-Delgado et al. 2010; van Dokkum 2005; van Dokkum et al. 2014). The detection of 
each class of collisional debris is essential since they trace the last merger events and store information about the mass assembly of their progenitors. In addition, their identification may modify their apparent morphology and then their classification in optical imaging surveys, such as the Sloan Digital Sky Survey (SDSS York et al. 2000).

The present work emphasizes a qualitative analysis of low surface brightness (LSB) fine structures identified in a numerical simulation conducted in a cosmological context. The first imaging observations of collisional debris to exhibit the distinctive shapes of tidal tails and stellar streams, mainly in early-type galaxies, were published in the Atlas of Peculiar Galaxies catalog (Arp 1966). In the case of shell structures, Malin \& Carter (1983) reported a catalog of 137 elliptical galaxies exhibiting shells or ripple-like structures. On the theoretical side, the first computational models of interacting galaxies revealed that the perturbations and debris are the outcome of gravity tides in disk galaxies (Toomre \& Toomre 1972). Since that time, substantial efforts undertaken to develop observational techniques aimed at identifying these substructures. The techniques include, for example, the unsharp masking and amplification of photographic images (Malin 1977, 1978), sky subtraction of individual images (Mihos et al. 2005), and extraction of structural components with GALFIT (Peng et al. 2002). Additionally, the application of galactic archeology also allows us to establish a demography of LSB structures in nearby galaxies (McConnachie et al. 2009; Crnojević et al. 2013), although its application is restricted to galaxies at a very low redshift due to spatial resolution and sensitivity.

The search for diffuse light was also a task carried out by amateur astronomers using simple cameras (e.g. Martínez-Delgado et al. 2009). Their long-exposure observations revealed spectacular images that later inspired the implementation of new techniques in conventional telescopes (Martínez-Delgado et al. 2010). Nonetheless, in spite of these innovative techniques, the detection of merger remnants continues to be problematic. Low spatial resolution or a limited field of view make it more difficult to recognize tenuous morphologies in the outer regions of galaxies. Perhaps the main issue is related to low surface brightness, expected to be below the level of $28 \mathrm{mag} \mathrm{arcsec}^{-2}$ and, most frequently, below $\sim 30 \mathrm{mag} \mathrm{arcsec}^{-2}$ (e.g. Johnston et al. 2008; Cooper et al. 2010). Thanks to the availability and advancement of deep imaging programs for nearby galaxies, it has been possible to detect the prominent structures that surround galaxies, with the large field of view provided by the mosaic camera (CFHT/MegaCam, NGVS, Ferrarese et al. 2012) or the specialized Dragonfly Telephoto Array, designed for LSB astronomy (van Dokkum et al. 2014).

In their pioneering work, Malin \& Carter (1983) revealed that numerous shells could be observed as tracers of interactions and mergers in 137 early-type galaxies. In the survey of 36 field galaxies aimed at detecting fine structures, Schweizer \& Ford (1985) identified $44 \%$ of weak shells and $\sim 10 \%$ of plumes and tidal tails. In more recent studies, Tal et al. (2009) present an optical image sample of 55 luminous elliptical galaxies and, by using a tidal interaction parameter, they find that $73 \%$ of these displayed tidal features. Meanwhile, Atkinson et al. (2013) report $\sim 26 \%$ of tidal features in their sample of 1781 galaxies from the CFHT Legacy Survey. On the other hand, Krajnović et al. (2011) identify $8 \%$ of tidal debris in a subsample of the ATLAS ${ }^{3 \mathrm{D}}$ sample of 260 early-type galaxies, whereas in the massive early-type galaxies of MATLAS deep imaging survey, it was found that $\sim 16 \%$ displayed streams and shell-like features, and $\sim 22 \%$ showed tails and plumes (Duc
2017). In the most recent studies, Hood et al. (2018) report an incidence of $17 \%$ of tidal features in the 1048 galaxies of the RESOLVE survey, as well as Kado-Fong et al. (2018), who identify $18 \%$ shell galaxies and $82 \%$ stellar stream systems from the subsample of 1201 galaxies of the SDSS spectroscopy images taken from the Hyper Suprime-Cam Subaru Strategic Program (HSC-SSP).

When it comes to numerical studies, only a few works have taken a census of tidal debris thus far. In the analysis of the hydrodynamical Illustris simulation, Pop et al. (2018) report that $18 \%$ of their massive galaxies display shell-like structures as a result of merger events with stellar mass ratios $\gtrsim 10: 1$. Karademir et al. (2019) explore a wide parameter space in mass ratios and relative orbits. They find that streams are formed by satellite infalling with a large angular momentum, while shells are the result of the radial infall of satellites with low angular momentum. More and more often, fine structures are being identified and classified through automatic techniques, such as those developed in Pawlik et al. (2016), Hendel et al. (2019), Walmsley et al. (2019). It is still necessary to identify fine structures visually in order to teach the machine how to improve the precision of image recognition and to verify procedures. In addition, different dark matter halo models should be explored since the fine structures will greatly depend on dark matter spatial distribution.

Several scenarios have been proposed to explain the morphology characteristics of tidal features. The disruption of merging galaxies may yield different features according to their relative orbits and the geometry of the encounter (Amorisco 2015; Hendel \& Johnston 2015). Analytical and numerical studies show that stellar shell structures are the remnants of disrupted satellites on near-radial orbits, while stellar streams are generated by near-circular orbits (Quinn 1984; Dupraz \& Combes 1986; Johnston et al. 2008; Karademir et al. 2019). Collisional debris is made up of transient structures that can last for short times or be disappear as a result of posterior interactions in a few Gyr (Stewart et al. 2008). Their recognition is primarily dependent on their surface brightness, in addition to several other factors, such as the type of substructure and orientation in the sky (Duc 2017). Thus, the determination of their survival timescale is crucial in reconstructing the merging history of galaxies, together with their numerical simulations. Until now, numerical works have been used to constrain these timescales. The earliest studies of semi-analytical models focus in the signatures of satellites orbiting the Milky Way. Johnston et al. (1999) reported a "preceding passage" of $\sim 0.7$ Gyr for tidal debris for the Sagittarius Dwarf Galaxy, demonstrating that the satellite is rapidly being disrupted and will only survive a few pericentric passages, that is, $1.3 \mathrm{Gyr}$. Hybrid models composed of semianalytical and N-body simulations of Milky Way-type stellar halos from Bullock \& Johnston (2005) estimate a median accretion time of $\sim 5 \mathrm{Gyr}$ for their satellite systems.

Hydrodynamical simulations of equal-mass mergers performed in isolated environments have determined several timescales based on different methods. Lotz et al. (2008) report an average timescale of $\sim 1.5 \mathrm{Gyr}$ using the Gini coefficient. Meanwhile, Ji et al. (2014) use visual inspection to estimate a merger-feature time (the moment when faint features disappear) of $\sim 1.38 \mathrm{Gyr}$ (for $\mu=25 \mathrm{mag} \operatorname{arcsec}^{-2}$ ), which is comparable to the timescale computed by Lotz et al. (2008). Current deep imaging surveys go much deeper and further studies are needed to estimate updated timescales.

The goal of the present work is to identify and classify the LSB features observed in a host halo based on the hydrodynamical simulation of Martig et al. (2009). These are 
zoom-in resimulations that reveal the fate of a typical massive galaxies in great detail after having undergone cold gas accretion and several mergers during a Hubble time. The cosmological context is taken from a previous large-scale simulation with reduced spatial resolution. The high resolution of the zoom-in simulation is capable of identifying most fine structures occurring at any epoch. In this analysis, five observers have visually classified a full mock catalog of stellar surface brightness maps (cf. the snapshots in the appendix). The aims of the classification are to: (i) characterize the shape of the faint stellar structures on the outskirts of the main central galaxy, (ii) take a census of the number of these structures and classify them into tidal tails, streams, and shells, (iii) to estimate the survival time of each substructure and study their dependence on the surface brightness limit and the projection on the sky plane.

In Sect. 2, we describe the numerical simulation carried out in a cosmological context (Sect. 2.1.1), the high-resolution resimulation (Sect. 2.1.2), and the phases of the galaxy evolution (Sect. 2.2). We describe the catalog of stellar surface brightness maps in Sect. 3. Results are reported in Sect. 4 and discussed in Sect. 5. Section 6 presents a summary of our conclusions.

\section{Numerical simulations}

\subsection{Simulation technique}

To analyze the galaxy evolution in the $\Lambda \mathrm{CDM}$ cosmological model, we use the numerical simulation of Martig et al. (2009). The approach demonstrates how the growth of a massive stellar spheroid through a series of mergers can be enough to stabilize a disk which is, by then, no longer self-gravitating, and therefore, quench star formation. Through this process, the galaxy becomes a red early-type object while continuing to accrete gas. The technique that was used consists of two steps: first a $\Lambda$ CDM cosmological simulation is run, with only dark matter, while the merger and accretion history for a given halo is extracted. In the second step, the mass assembly history is re-simulated at a higher resolution, replacing each halo with a realistic galaxy, containing gas, stars, and dark matter.

\subsubsection{Cosmological simulation}

The cosmological simulation used to carry out this study was performed with the RAMSES code (Teyssier 2002), based on the Adaptive Mesh Refinement (AMR) technique. The box has a comoving length of $20 \mathrm{~h}^{-1} \mathrm{Mpc}$, and contains $512^{3}$ dark matter particles with a mass resolution of $6.9 \times 10^{6} M_{\odot}$. The dark matter halos are identified using the HOP algorithm of Eisenstein \& Hut (1998), and the merger and accretion histories are extracted following the main halo from $z=2$ to $z=0$. All the halos (of the merger history) and diffuse particles (of the accretion history) that cross a fixed spherical boundary drawn around the target halo are recorded. The sphere is larger than the initial main halo at $z=2$ and encompass satellites, as well as "diffuse" particles, that is, those that don't belong to any halo. The second step zoom-in resimulations were carried out for Milky Waymass galaxies in low density environments (Martig et al. 2012). Within this sample, we selected a case where there is a major merger with a post-merger early-type phase galaxy. There is no particular selection bias, nor were the galaxies selected on the basis of an abundance of fine substructures.

\subsubsection{High-resolution resimulation}

The next step consisted of a high-resolution resimulation using a particle-mesh code described in Bournaud \& Combes (2002,
2003), in which the gas dynamics is modeled using a sticky particle scheme. The maximal spatial resolution is $130 \mathrm{pc}$ and the mass resolution is $1.4 \times 10^{5} M_{\odot}$ for stellar particles initially present in the galaxies, $2.1 \times 10^{4} M_{\odot}$ for gas and stellar particles formed during the simulation, and $4.4 \times 10^{5} M_{\odot}$ for dark matter particles. Star formation is computed following a SchmidtKennicutt Law (Kennicutt 1998), where the SFR is proportional to the gas density to the exponent of 1.5 , setting a threshold for star formation at $0.003 M_{\odot} \mathrm{pc}^{-3}$. The simulation does not include supernova explosions, nor AGN feedback.

The resimulation starts at redshift $z=2$ and evolves down to $z=0$, including all the halos and the "diffuse" particles embedded in the spherical boundary around the target halo, recording their mass, position, velocity and spin. Each halo is replaced by a realistic galaxy made of gas, stars and dark matter particles, and by replacing each diffuse particle with a blob of lower-mass, higher-resolution gas and dark matter particles (see Appendix A of Martig et al. 2009 for more details). Each small halo starts to interact with the main halo following the orbital and spin parameters given at the beginning of the cosmological simulation (e.g., Fig. 2 and subsequent figures).

\subsection{Merger history and stellar mass growth of the studied galaxy}

The central galaxy studied in this simulation has a mass similar to that of the Milky Way. Its corresponding host halo does not belong to a rich group or a galaxy cluster as most early-type galaxies (ETGs) in the MATLAS survey (see snapshots in the appendix for the galaxy environment). During the simulation, the host halo increases from a mass of $2 \times 10^{11} M_{\odot}$ at $z=2$ to $1.4 \times 10^{12} M_{\odot}$ at $z=0$. The galaxy evolution can be decomposed into 3 main phases (see the plot 3 of Martig et al. 2009). The simulation starts out with a gas-rich disk galaxy and an intense phase of minor mergers with a mass ratio between 1:4 and 1:10. This period lasts from $z=2$ to $z \sim 1$. Afterwards, a quiet phase takes place between $z \simeq 1$ and $z \simeq 0.2$. This is a quiescent period studied by Martig et al. (2009) in which star formation is suppressed. Over this period, there is no major event occurring, however, diffuse gas is continuously being accreted. In the final phase, the galaxy undergoes a major merger event, along with an increase in the diffuse gas accretion rate, which lasts from $z=0.2$ to $z=0$. These three phases are representative of any merging history in the life of a galaxy and, thus, our estimations of life-time of fine structures will be statistically significant.

\section{Surface brightness maps}

\subsection{Mock image generation}

We use the models of Bruzual \& Charlot (2003; BC03) to compute mock images of the stars in the simulation. We follow the technique developed in Michel-Dansac \& Wozniak (2004). We briefly describe how the mock images are made. For all stellar particles in the simulation, we compute the stellar spectra with a linear interpolation of age in the BC03 tables. For this, we use tables based on a Salpeter IMF (Salpeter 1955). We assume a solar metallicity for the stellar particles. The simulation does not follow the chemical evolution. For one projection, particles are projected on a 2D grid using a Cloud in Cell (CIC) algorithm (Birdsall \& Fuss 1969). Then, for each pixel we sum up the spectra of individual particles to get the composite spectrum. These spectra are finally integrated in the CFHT/MegaCam photometric filters in order to produce surface brightness maps. 


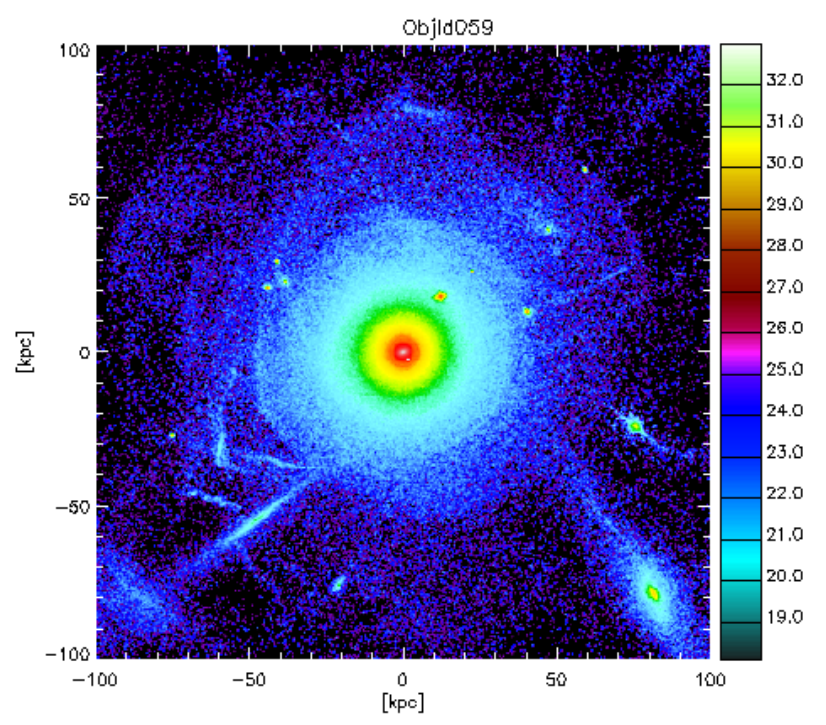

Objld059

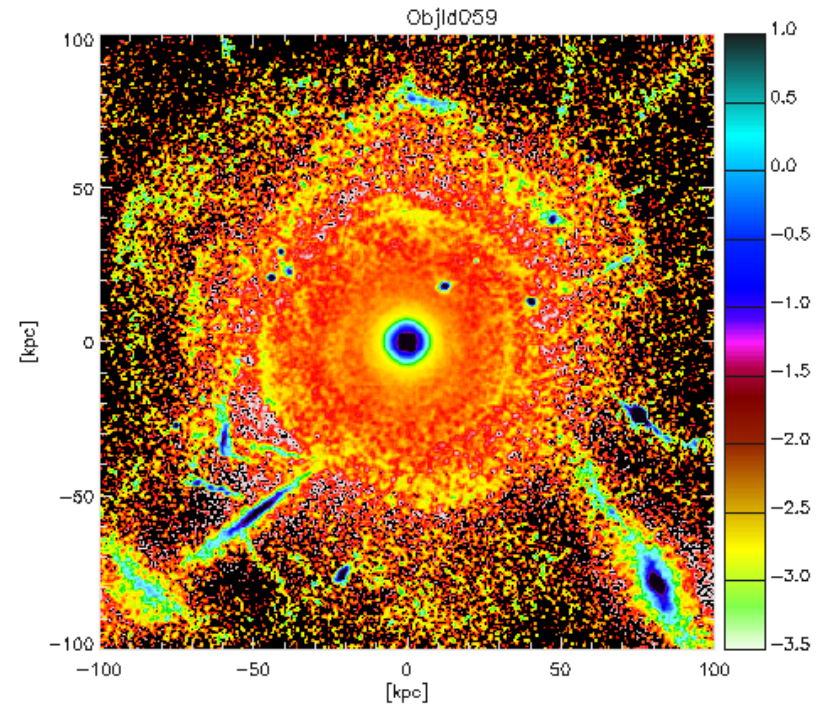

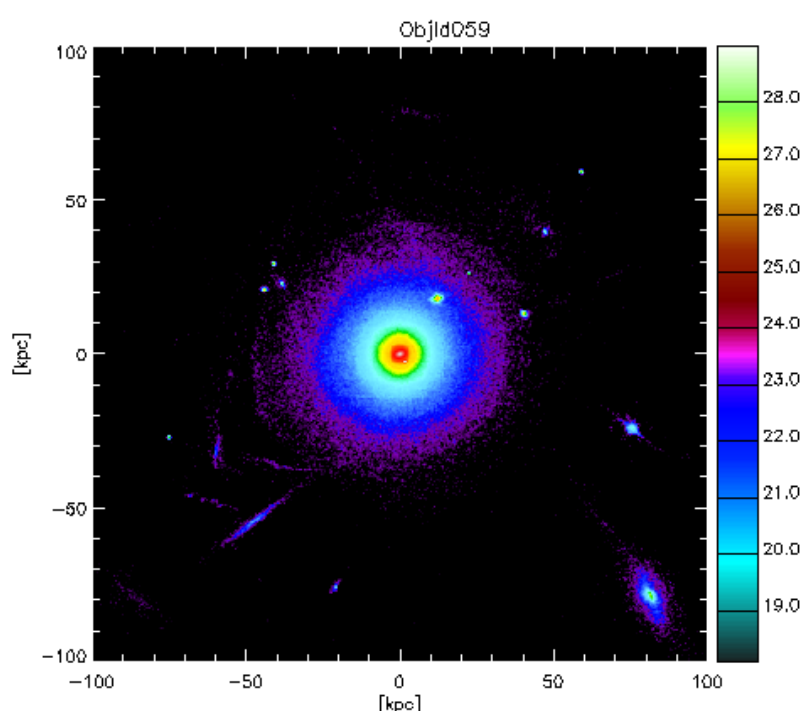

Objld059

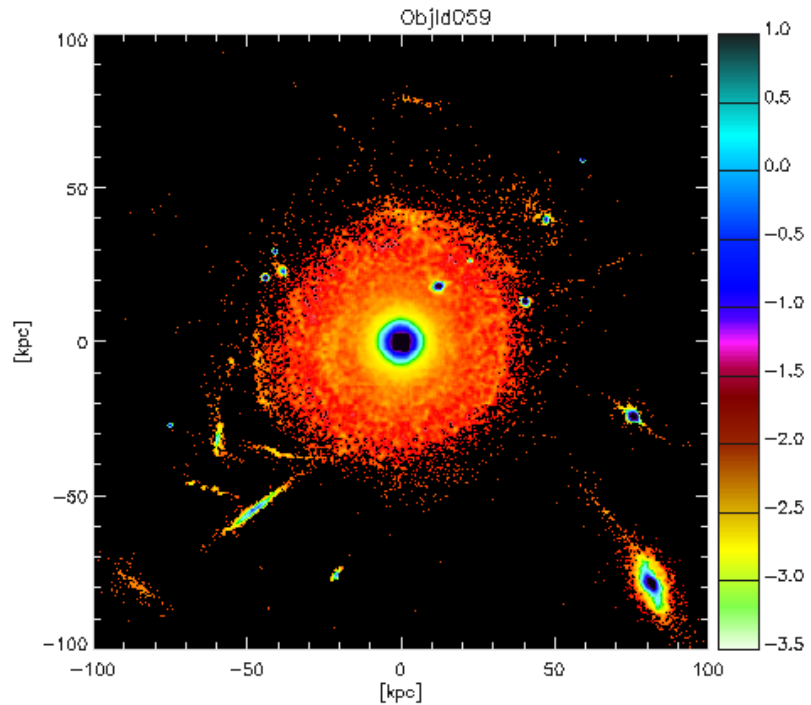

Fig. 1. Surface brightness map of central galaxy shown in $g$-band with two different color scales (resp., upper and lower panels) and with cut at $33 \mathrm{mag} \operatorname{arcsec}^{-2}$ (left panels) and $29 \mathrm{mag} \operatorname{arcsec}^{-2}$ (right panels).

We have compiled a catalog of images for the 35 snapshots of the simulation and the three projections, presenting a total of 105 images. For each image, we prepared two $g$ band maps: one with a cut at $33 \mathrm{mag} \operatorname{arcsec}^{-2}$ (Valls-Gabaud 2017) and the other at $29 \mathrm{mag} \operatorname{arcsec}^{-2}$. The latter value corresponds to the surface brightness limit of the MATLAS survey. We also added unsharp masked maps, a technique that has proven very efficient in enhancing LSB substructures. The first snapshot starts at $3.5 \mathrm{Gyr}$ and the last one ends at $13.7 \mathrm{Gyr}$ (in addition to an extra simulation which evolved until $15.7 \mathrm{Gyr}$ ). The time interval is $0.3 \mathrm{Gyr}$. Below, we provide a phenomenological description of collisional debris, based on the observational work of Duc et al. (2015) (see e.g., Fig. 1). To visualize tidal streams, tails and shells, we believe that dust extinction is not significant because the fine-structure features are located in the outer parts of galaxies, where there is little gas or dust.

\subsection{Visual classification}

For the visual identification, we proceeded in a similar way to earlier observational surveys aimed at identifying substructures optically (e.g. Tal et al. 2009; Atkinson et al. 2013; Duc et al. 2015; Duc 2017). A team of five members carried out a visual inspection of each mock image. We identified and classified the fine structures that were observed and took a census of them for both surface brightness cuts. Note that all the images (various snapshots and projections) in the catalog have been randomly ordered for the classification in order to avoid two close snapshots in the same projection following the other in the catalog or two projections of the same snapshot. This ensures that our classification is not influenced by the stellar structures of recent history or another viewing angle. Indeed, in real observations, there is no equivalent of two consecutive snapshots of the same merger event. In our case, each image is initially considered entirely independently of any other. This also allows us to verify the consistency of the method once the merger history is reconstructed.

Figures 2-4 demonstrate three examples of tidal tails, stellar streams, and shells, respectively, each projected in two directions, and based on the case of the lowest surface brightness limit, that is, $\mu=33 \mathrm{mag} \operatorname{arcsec}^{-2}$. Below, we define the substructures categorized within the three morphologies mentioned above. 

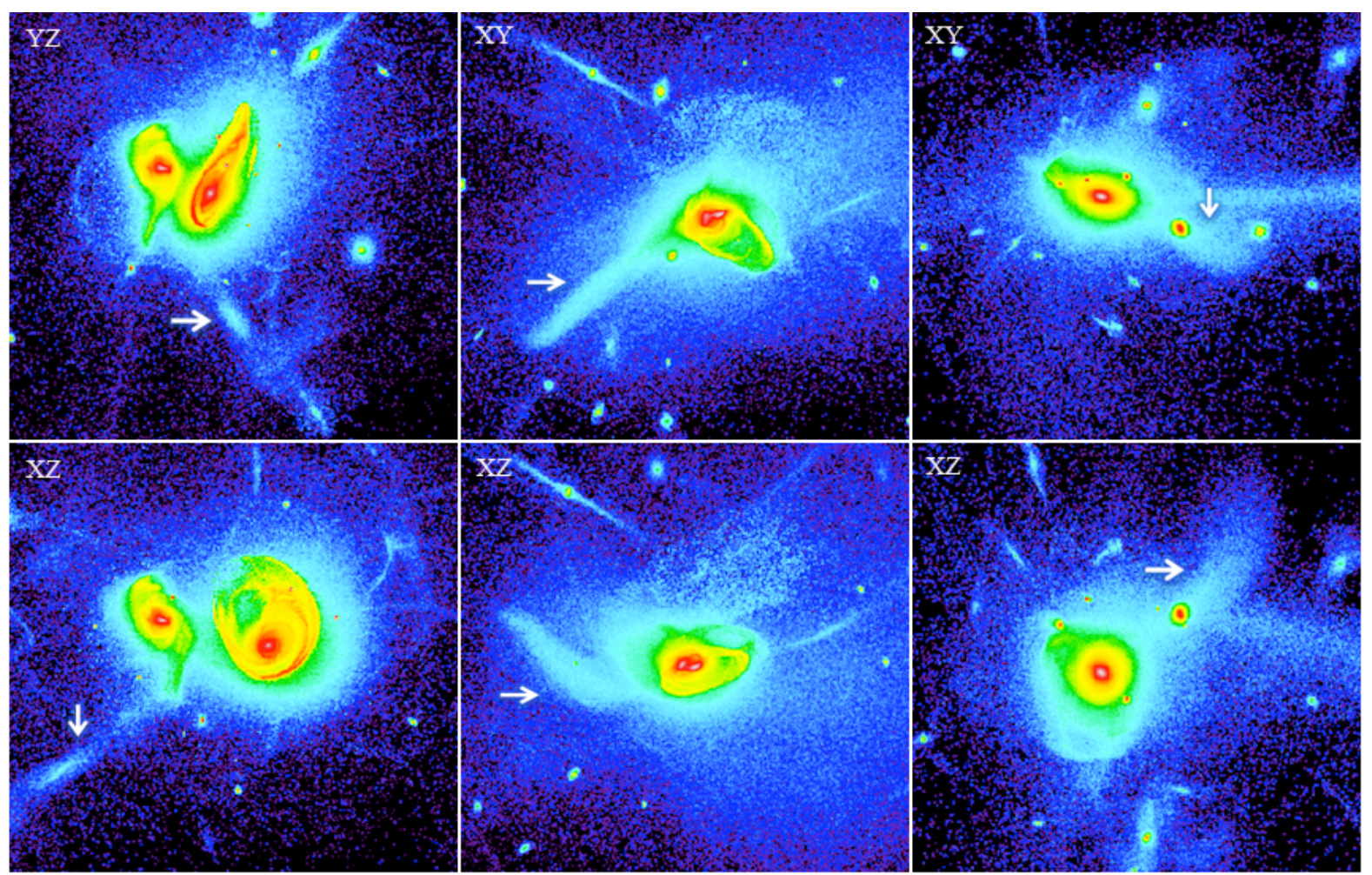

Fig. 2. Examples of interactions between central galaxy and its nearby companions, which demonstrate protuberant tidal tails (indicated with a white arrow) for three different objects (resp., right, middle and left panels) in two random projections: XY in the upper panel and XZ at the bottom.

Tidal tails are thick, radially elongated structures that are connected to the host galaxy. The visual impression is that the tidal tail appears to be emanating from the galaxy. In the case of any confusion between tail and stream, we relied on the aspect of thinness to distinguish between streams and tail; tails being thicker than streams. The thickness separation between the two is of the order of $300 \mathrm{pc}$. Over time, the dilution of tidal tails implies a widening, so there is no confusion.

Stellar streams are thin, elongated stellar structures which look like narrow, long filaments. They can be associated with galaxy satellites, exhibiting the known S-shaped structures that emanate from the main galaxy and characterize satellite disruption. But this is not a requisite, so they might also not be associated clearly with any galaxy, or physically associated with the central galaxy.

Shells have circular concentric shapes and are sharp-edge arc structures. Depending on their intrinsic nature and their projection, they appear to be aligned with a common axis or randomly spread around the central galaxy. When aligned to the major axis of the host, they are interleaved, meaning that they appear to accumulate alternatively on each side of the galaxy. This stellar accumulation can be associated with successive apocenters of an oscillating radial orbit following a satellite accretion event. When they extend to larger radii, they become more diffused.

To illustrate the three kinds of fine structures, we compare typical examples for each, in both observations and simulations, in Fig. 5.

\section{Results}

\subsection{Evolution of stellar mass}

The stellar mass of the central galaxy is plotted as a function of time in Fig. 6. We distinguish the provenance of the stellar mass into two categories. First, we show the contribution of the in situ mass, referring to the stars born inside of the main galaxy. Secondly, the ex situ mass, or accreted mass referring to the stars formed in the satellite galaxies and accreted afterwards into the main galaxy. We note that since the simulation starts at $z=2$ with preprepared galaxy models, a third contribution to stellar mass is the initial mass of the galaxy. These three phases describe the evolution of the main galaxy. In addition, at larger scales, a number of satellites orbit the halo all over the simulation. In Table 1, we describe the properties of the most important satellites that interact with the main galaxy during the simulation. The merging satellites are identified using an arbitrary number. Their merging time, their stellar mass, and their mass ratio with respect to the main galaxy are all listed.

\subsection{Identified substructures}

Having defined the method and described the mock catalog of surface brightness images, we explore our findings and the substructure properties observed in this analysis.

In Figs. 7-9, we present the time evolution of the number of tidal tails, stellar streams and shells, respectively, for three different projections, $x-y, x-z$ and $y-z$ (as indicated in each panel). Time begins at the start of the resimulation $(z=2)$, and continues after the present time $(z=0)$, so that the future of fine structures can be estimated. The panels display the curves of the average value from the five team members who made the visual inspection for each time interval (snapshot) and their respective scatter. We present this census for both surface brightness limits $\mu=29 \mathrm{mag} \operatorname{arcsec}^{-2}$ ("detected") and $\mu=33 \mathrm{mag} \mathrm{arcsec}^{-2}$ ("total"), respectively.

In addition, in order to analyze the correlation between the generation of features and the presence of mergers, in Fig. 10 we 


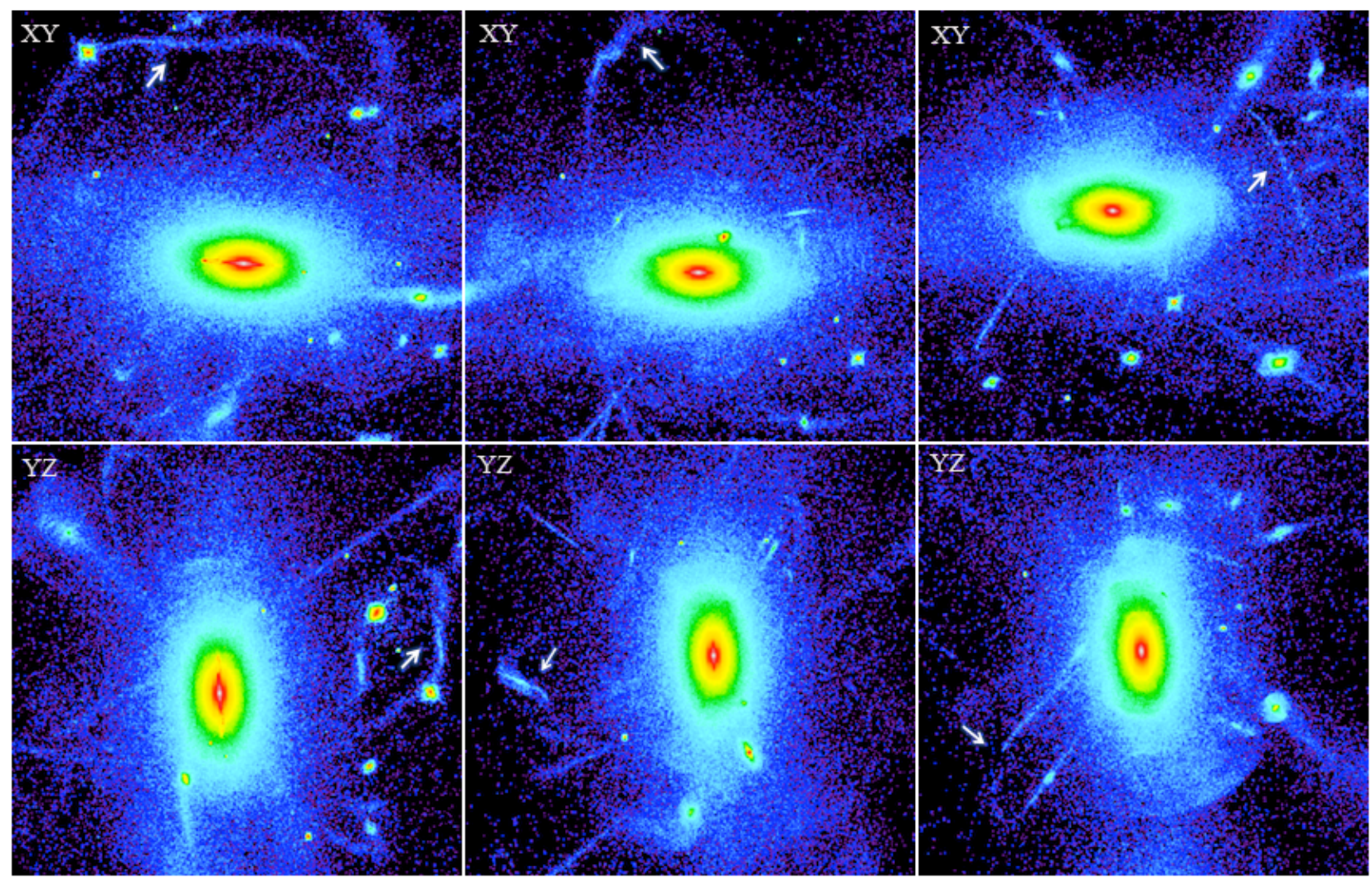

Fig. 3. Examples of central galaxy hosting disrupted satellites based on presence of prominent stellar streams (indicated with a white arrow) for three different objects (resp., right, middle and left panels) in two random projections: XY in the upper panel, and $\mathrm{YZ}$ at the bottom.

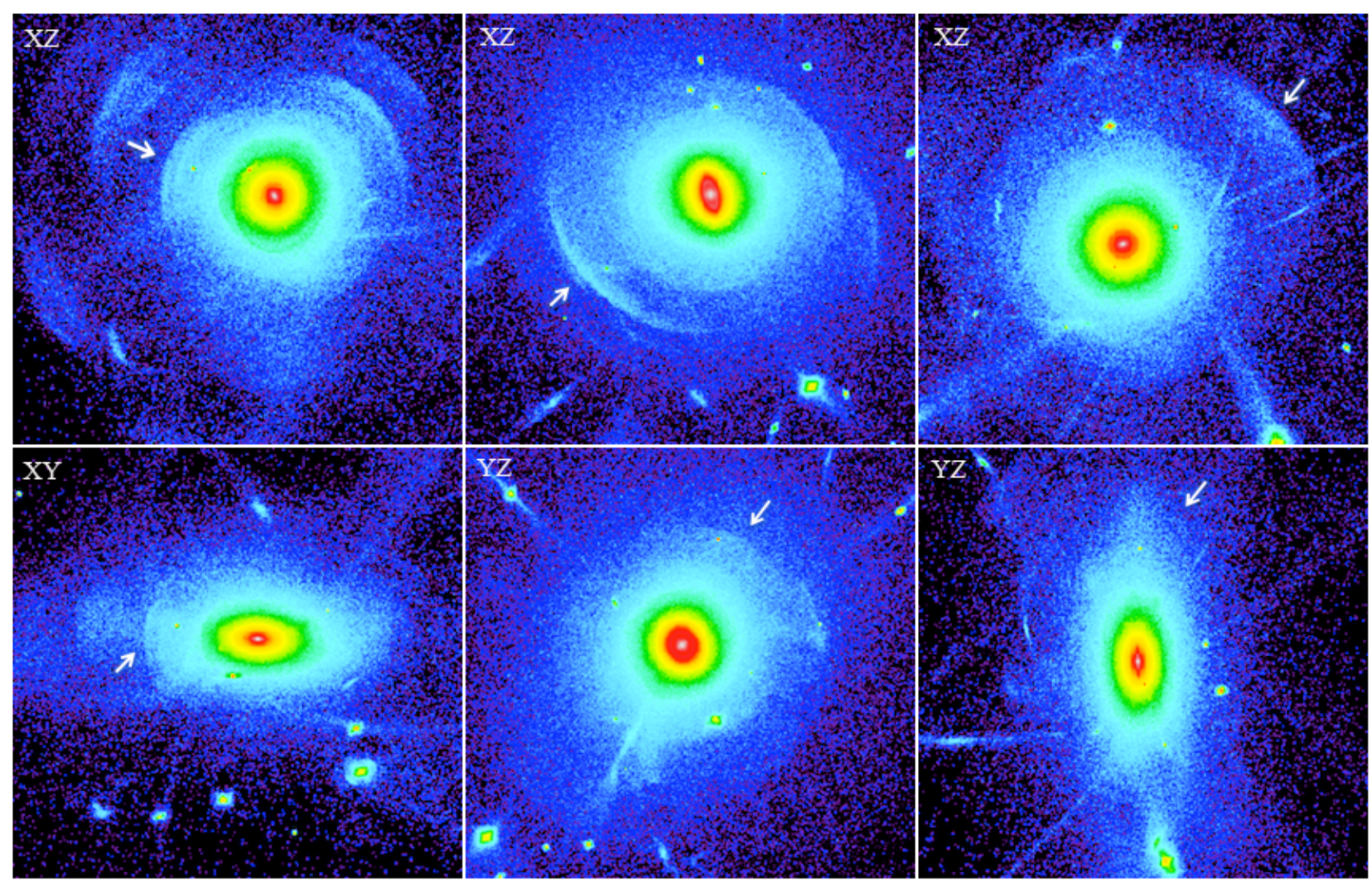

Fig. 4. Examples of central galaxy displaying several shells around it (indicated by white arrow) for three different objects (resp., right, middle and left panels) in three random projections: $\mathrm{XZ}$ in the upper panel, $\mathrm{XY}$ and $\mathrm{YZ}$ at the bottom. 


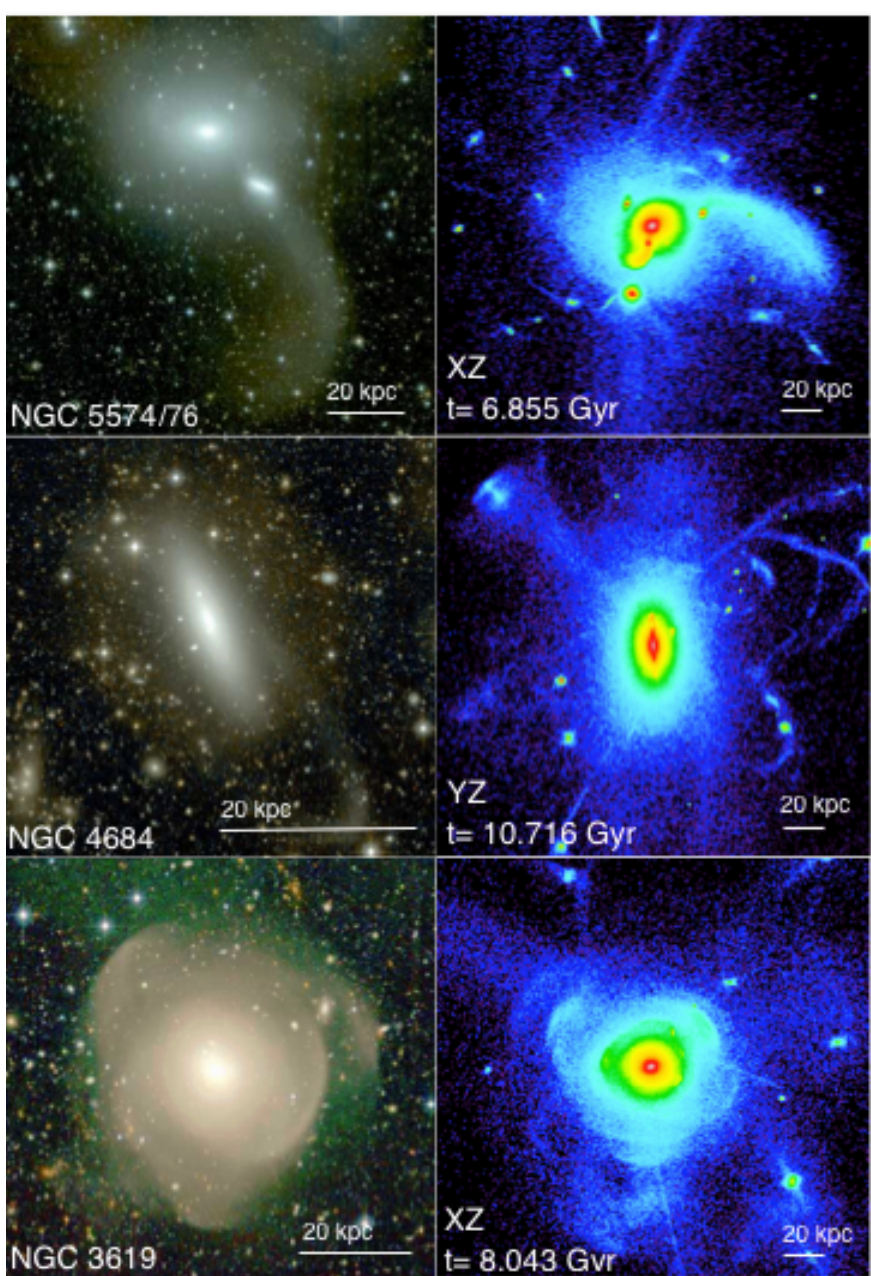

Fig. 5. Comparison of LSB substructures around observed and simulated galaxies. Left panels: examples of ETGs from MATLAS Survey. From top to bottom: system NGC 5574/76 (tidal tails emanating from NGC 5576), stellar stream in NGC 4684 and interleaved shells in NGC 3619. Right panels: examples of mock surface brightness images exhibiting prominent tidal tails, streams, and shells at different times of simulation in random projections. Note the white scale bar indicating $20 \mathrm{kpc}$ : the scales are comparable across observations and simulations.

show the accreted stellar mass (red line in upper panel) and we use arrows to indicate where the mergers take place. We point out the most significant mergers and their mass ratios with red numbers. In the lower panel, we present the corresponding evolution of each fine structure. Here we plot the highest numbers of features over the three projections for tidal (blue dotted line), streams (green dotted and dashed line) and shells (black dashed line). The identification of each substructure detected during the formation of the galaxy corresponds to the time period of $t=3.5 \mathrm{Gyr}(z=2)$ to $t=16 \mathrm{Gyr}(z>0)$. The major merger at $T=11.5 \mathrm{Gyr}$ is destroying most of the previous fine-structures; shells in particular. Some more shells and tails are rebuilt after that at the same rate as before. It will take some time to reach the same level of fine structures as was reached in the quiet phase.

We note that the curves indicating the number of fine structures as a function of time (Figs. 7-9) are reconstructed and reordered afterwards. It is remarkable that they make sense, that is, the correlations between individual consecutive snapshots as visible and as expected. Below, we describe the main characteristics of the census of tidal debris.

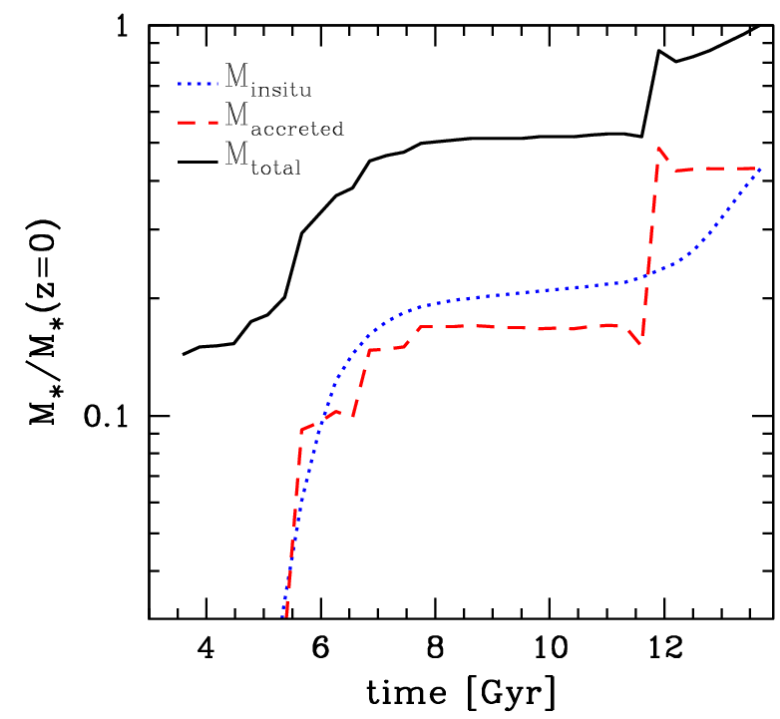

Fig. 6. Evolution of stellar mass for main galaxy as function of time. The total stellar mass (black solid line) has been decomposed into the in situ mass (blue dotted line), that is, stars formed in the main galaxy, and accreted mass (red dashed line), that is, stars formed in a companion galaxy and then accreted into the main galaxy.

Table 1. Stellar mass ratio of main mergers.

\begin{tabular}{lcc}
\hline \hline$t_{\text {merger }}[\mathrm{Gyr}]$ & $M_{\star}\left[M_{\odot}\right]$ & Stellar mass ratio \\
\hline 4.77 & $4.4 \times 10^{9}$ & $0.14(7: 1)$ \\
5.67 & $13.0 \times 10^{9}$ & $0.31(3: 1)$ \\
6.26 & $1.5 \times 10^{9}$ & $0.04(25: 1)$ \\
7.20 & $12.0 \times 10^{9}$ & $0.17(6: 1)$ \\
7.75 & $5.0 \times 10^{9}$ & $0.06(16: 1)$ \\
11.9 & $74.0 \times 10^{9}$ & $0.67(1.5: 1)$ \\
\hline
\end{tabular}

Notes. Stellar mass ratio has been measured just before galaxy merger.

\subsection{Formation mechanism and survival time}

The formation scenario for each substructure is the first bit of information we can infer from the evolution of the simulation. We know by construction all the merger events of the central galaxy in all its evolution phases. The counting of the substructures is, thus, a direct outcome and it allows us to draw correlations between individual substructures and the corresponding merger event. In Fig. 10, we represent the correlation between the generation of features and the occurrence of mergers.

\subsubsection{Tidal tails}

We find that tails are produced during the first and the third phase of galaxy evolution as a result of tidal forces acting within the host galaxy. In the first phase, we observe an average of two tails associated with the intermediate-mass merger and major merger events (mass ratios between 7:1 and 3:1). In the second phase, their detection drastically decreases due to the lack of any kind of mergers. In the last phase, a peak of incidence appears when the major merger (mass ratio of 1.5:1) takes place. Prominent tidal tails can be evaluated through visual inspection at $6.8 \mathrm{Gyr}$ and $11.9 \mathrm{Gyr}$ in the snapshot evolution (see Figs. A.1-A.3). We estimate a survival time of $\sim 2 \mathrm{Gyr}$. 


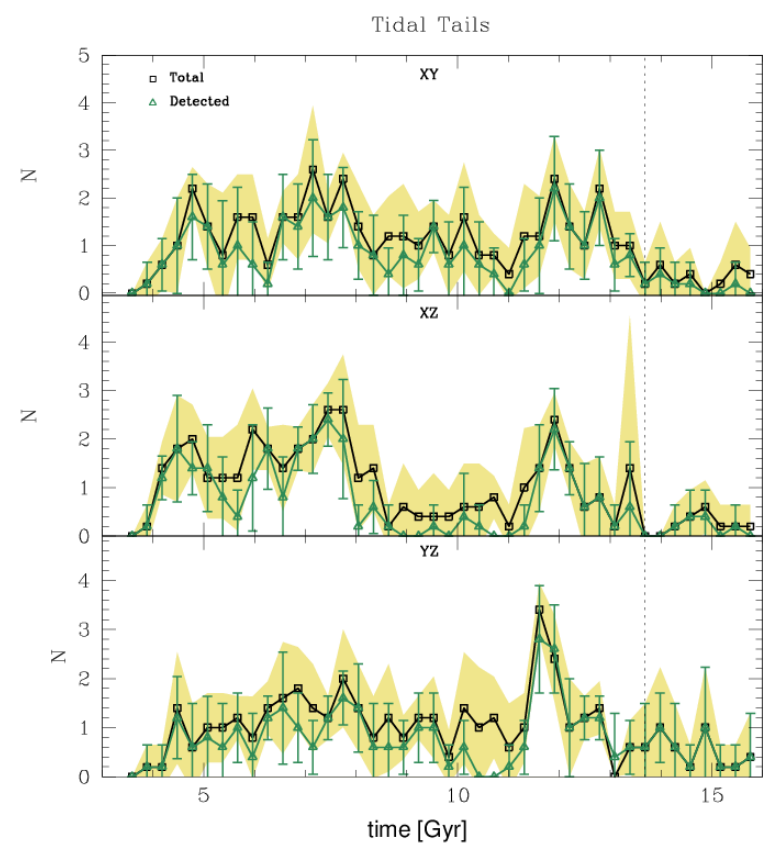

Fig. 7. Evolution of tidal tail numbers. The three panels show the results for the three projections (XY, XZ, and YZ). In each panel, the black lines and the yellow shaded area represent the total number of tidal tails and their respective scatter, whereas the green ones are the number of tidal tails that are detectable on images with a cut in surface brightness at $29 \mathrm{mag} \mathrm{arcsec}^{-2}$. The time $(t=0)$ corresponds to the Big-Bang, and the re-simulation is longer than a Hubble time, beyond $z=0$, to estimate the future of fine structures.

\subsubsection{Stellar streams}

Streams are the most frequent fine structure features in our simulation (an average of $\sim 8$ streams detected through visual inspection, see Fig. 8). They appear in the first series of consecutive minor mergers, increasing in number over time, although some of them are destroyed when the following merger events occur. The highest peak of incidence is reached during the quiescent phase (second phase). The absence of important mergers during this phase allows for the increase in the production of streams and their survival time by the lack of processes to that would eliminate them. They trace the initial orbit of the satellites which are disrupted during this quiet phase, producing long, extended narrow streams. We observe a decrease in the detection number when the major merger appears and eliminates many of them in the last phase of the simulation. Under all the physical conditions explained above, we derive a life time of $\sim 3$ Gyr.

The stellar streams are present during the quiescent phase of the galaxy, and even demonstrate their peak in this period, while there is no minor merger (nor major one) in all this phase. This may look paradoxical. Either the stellar streams develop from the minor mergers coalesced in the previous phase, or there is another mechanism to form these streams. The latter could be due to the accretion of cold gas (e.g. Bournaud et al. 2005).

\subsubsection{Shells}

In our simulation, shell formation is associated with both intermediate-mass mergers and major mergers. Previous works have shown they are commonly related with intermediate-mass mergers (e.g. Pop et al. 2018; Karademir et al. 2019). The largest generation of shells is visible during the quiet phase, just after

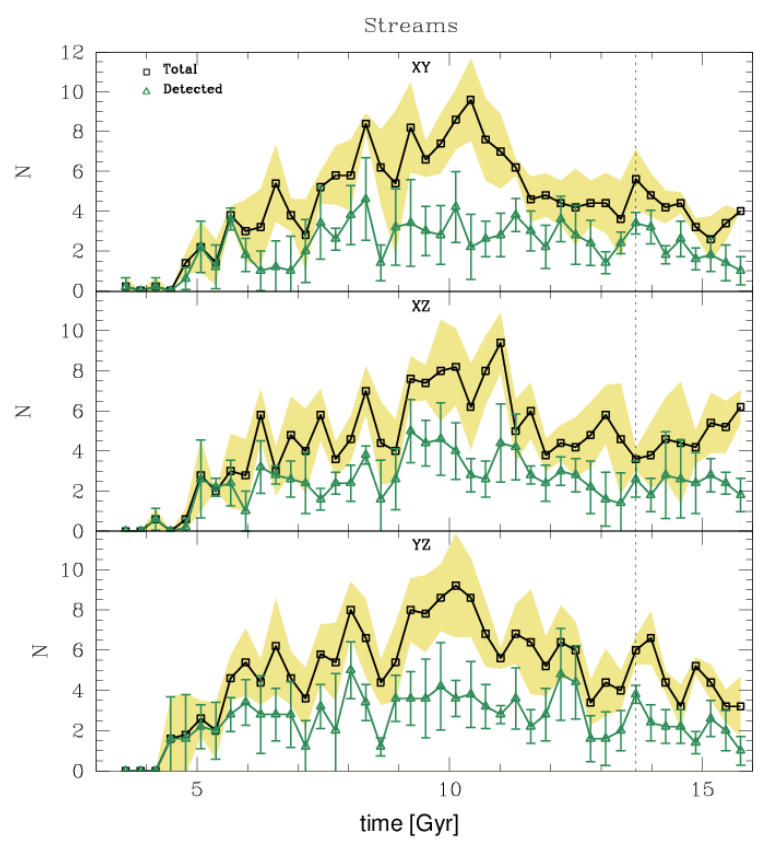

Fig. 8. Evolution of stellar stream numbers. Three panels show results for three projections. In each panel, the black lines and the yellow shaded area are the total number of stellar streams and their respective scatter, whereas the green ones represent the number of stellar streams that are detectable on images with a cut in surface brightness at $29 \mathrm{mag} \operatorname{arcsec}^{-2}$. The time $(t=0)$ corresponds to the Big-Bang.

the first phase of mergers has occurred, whereas a second peak of shells is produced just after the major merger takes place. We attribute this high incidence of shells to the same phenomenon as in the case of streams, that is: when there are no violent processes to eliminate them, they remain numerous in the quiet phase. Following the snapshot evolution (see Fig. A.1), we note that once the corresponding satellite falls into the central potential, shells appear and acquire their known arc-like shape. Initially, they are spread around the galaxy at small distances from the center and are distributed in an interleaved way. They accumulate near the apocenters of their orbits, and develop progressively to the outskirts of the central galaxy. Consequently, due to their density wave nature, they propagate towards larger and larger distances, and their numbers increase. According to the duration of their incidence peak, that is, from the width of the curves in Fig. 9, and assuming that no shell is produced during the quiet phase, we estimate a lifetime of $\sim 4 \mathrm{Gyr}$.

\subsection{Sensitivity on the surface brightness cut}

The surface brightness limit is one of the main issues relevant to the identification of fine features, according to observational studies. Traditional images from the SDSS survey (York et al. 2000) reach a value of $26.4 \mathrm{mag} \operatorname{arcsec}^{-2}$ in the $g$-band. In the sample from CFHT Legacy Survey (Atkinson et al. 2013) and the observations with the MegaCam of Tal et al. (2009), also in the $g$-band, they barely detect substructures at $\sim 28 \mathrm{mag} \mathrm{arcsec}^{-2}$. Deeper observations like the LSB-optimized NGVS Survey (Ferrarese et al. 2012), the ETG from ATLAS ${ }^{3 D}$ (Duc et al. 2015), and the Large Program MATLAS Survey (Duc 2017) achieve a value of 29 mag arcsec${ }^{-2}$. These values relate to the local surface brightness. However, it is possible to define integrated-light surface brightness, and a very low level of scattered light (one order of magnitude below the best-performing 


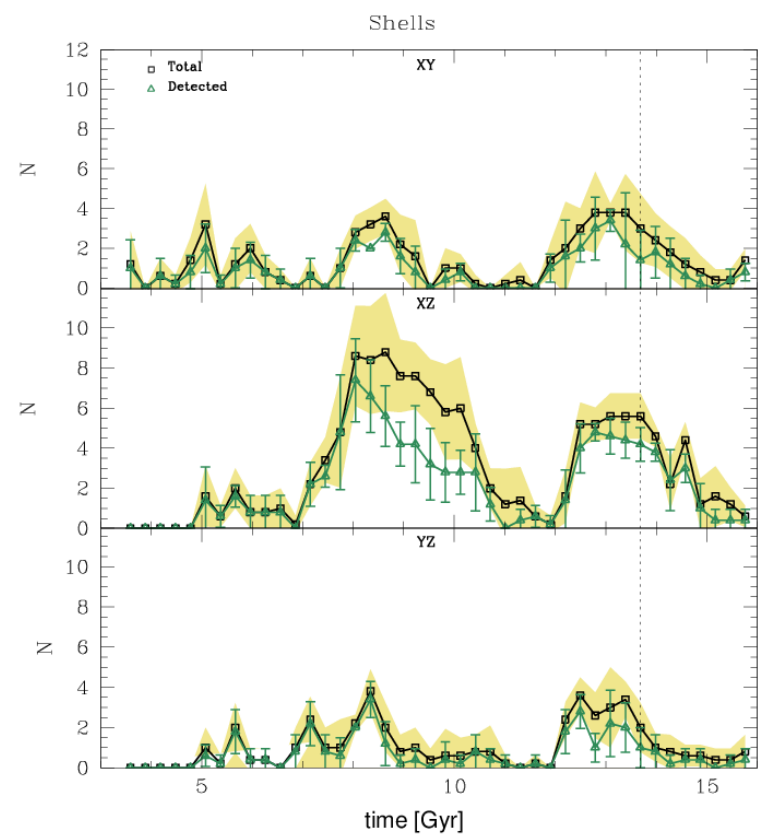

Fig. 9. Evolution of shell numbers. Three panels show results for three projections. In each panel, the black lines and the yellow shaded area are the total number of shells and their respective scatter, whereas the green ones represent the number of shells that are detectable on images with a cut in surface brightness at 29 mag $\operatorname{arcsec}^{-2}$.

telescopes), with the lowest limit achieved with the Dragonfly Telephoto Array (van Dokkum et al. 2014), having a value of $32 \mathrm{mag} \operatorname{arcsec}^{-2}$. The latter reveals spectacular hidden substructures in the external regions of galaxies. These surface brightness limits could be reached locally by dedicated space-based missions, such as the Messier project (Valls-Gabaud 2017).

On the simulation side, there is the freedom to reproduce deep images with low surface brightness. The values range from $28 \mathrm{mag} \mathrm{arcsec}{ }^{-2}$ (Ji et al. 2014) to even $38 \mathrm{mag} \mathrm{arcsec}^{-2}$ (Bullock \& Johnston 2005; Johnston et al. 2008). Motivated by all these previous studies on the detectability of faint features in merger remnants, the present study selects two values of the surface brightness limits, $\mu=29 \mathrm{mag} \mathrm{arcsec}^{-2}$ and $\mu=33$ mag $\operatorname{arcsec}^{-2}$, respectively, to take a census of LSB substructures.

\subsubsection{Tidal tails}

We do not find any substantial dependency on the surface brightness for this type of substructure. We detect a comparable number of tidal tails for both surface brightness cuts per time step (snapshot). The curves in the three panels of Fig. 7 follow the same trend over the course of the evolution. We attribute this result to their prominent nature, which is a result of major interactions and, therefore, fall above the detection threshold.

\subsubsection{Stellar streams}

We find a high dependence on the surface brightness limit for this type of substructure. As seen in the three panels of Fig. 8, the incidence of streams is considerably higher for $\mu=33$ mag $\operatorname{arcsec}^{-2}$ than for $\mu=29$ mag $\operatorname{arcsec}^{-2}$. The peak of incidence reaches an average number of 9 streams for the first cut, while for the second cut, the peak only reaches 4 streams. Streams are tracers of minor mergers and, hence, they have a

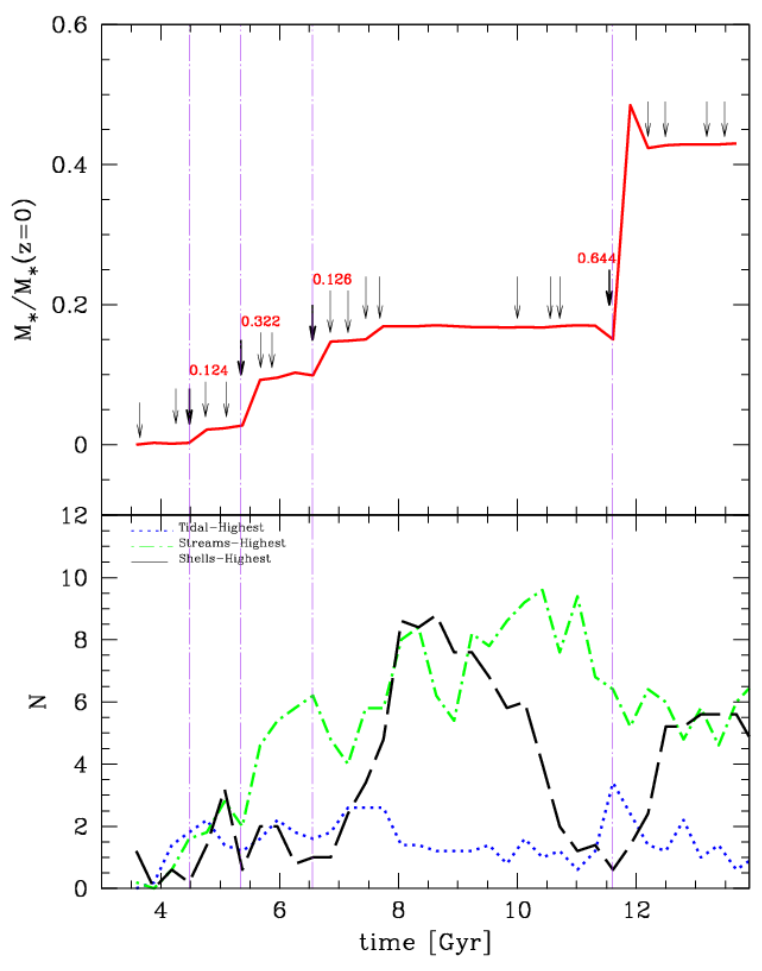

Fig. 10. Comparison between accreted stellar mass and all types of fine structures. Upper panel: red line represents evolution of accreted mass. Arrows indicate when mergers occur. Red numbers over bold arrows correspond to intermediate mass and major mergers. Lower panel: evolution of highest value of three projections in each fine structure. Blue dotted line is for tidal tails, green dotted and dashed line is for streams and black dashed line is for shells. Purple dotted and dashed lines in both panels indicate the correlation between main mergers and substructures.

narrow morphology, making their detection difficult. They are faint, but as they are more narrow, they may be identifiable for longer than the more diffuse tails. Figure 11 presents the surface brightness map of a particular stream, under the two cuts, to illustrate a clear example of this difference. With the low level of surface brightness (left panel), a wide variety of extended streams surround the central galaxy, along with the tail of a prominent satellite, in the higher surface brightness cut (right panel), and only the central regions of the whole system are seen. Stellar streams are the type of substructure which present the strongest dependency on surface brightness in comparison with tails and shells.

\subsubsection{Shells}

Shells are slightly sensitive to surface brightness. A significant difference is observable between the maximal peaks of the two curves in the middle panel of Fig. 9 (projection $y-z$ ). For this peak, we detect, on average, two shells more for the case of $\mu=33 \mathrm{mag} \mathrm{arcsec}^{-2}$ than for $\mu=29 \mathrm{mag} \mathrm{arcsec}^{-2}$.

\subsection{Dependence on the projection}

Another important issue relevant to the identification of substructures using visual inspection is the effect of the projection. Fortunately, our approach reproduces three projections for each snapshot in order to consider the effects of the sky orientation on each class of substructures. 

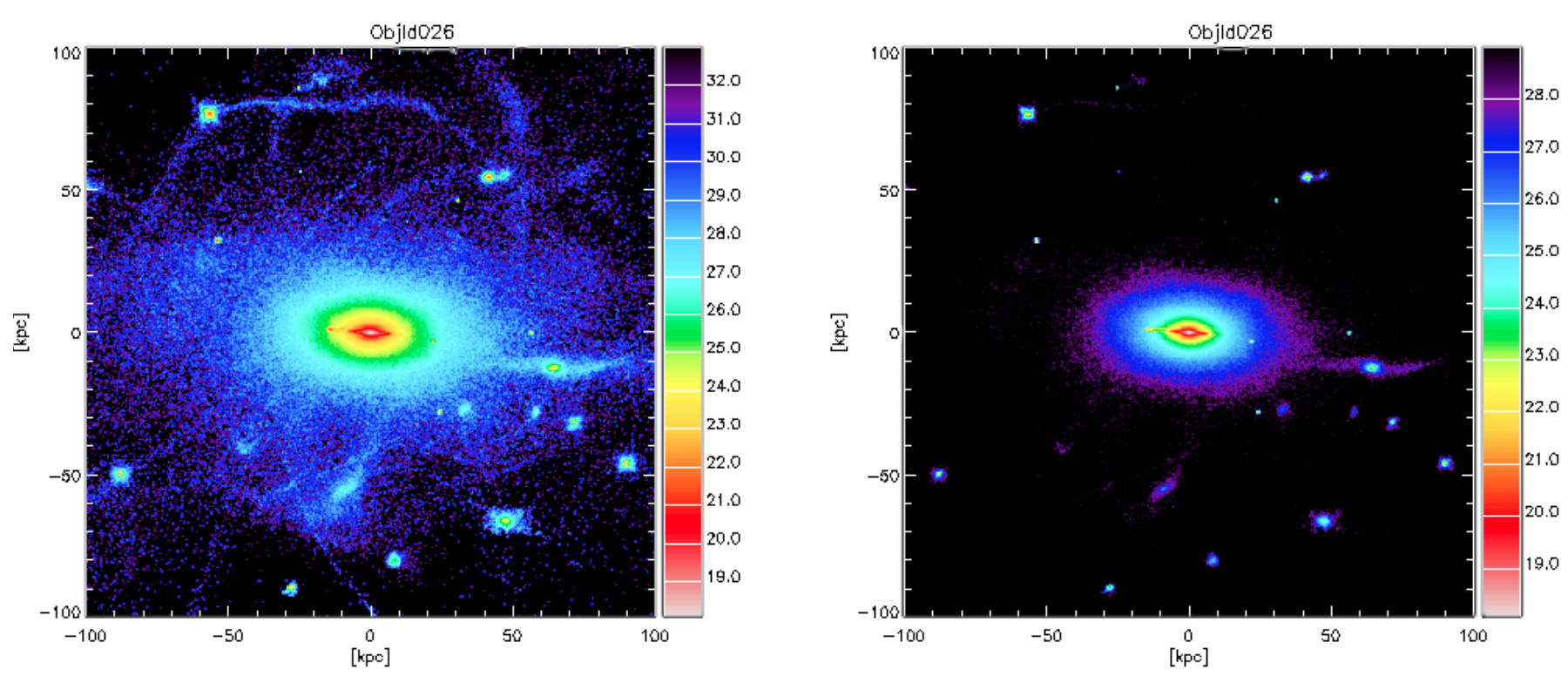

Fig. 11. Example of stellar stream appearance with a cut at $33 \mathrm{mag} \operatorname{arcsec}^{-2}$ (left panel) and 29 mag $\operatorname{arcsec}^{-2}$ (right panel).

\subsubsection{Tidal tails}

with the phrase in bold as this format amounts to the equivalent of bullet points. We do not find any dependence on the projection. We compare the three projections in the corresponding panels of Fig. 7 and we find a similar tendency for the curves across all periods of time. The same number of tidal tails can be detected in any projection of the simulation. We attribute this behavior to the $3 \mathrm{D}$ nature of tails when encounters are not coplanar.

\subsubsection{Stellar streams}

Streams do not exhibit dependence on the orientation. They are visible in all directions. The same number of streams can be detected in any projection. Curves in the three panels of Fig. 8 follow the same trend.

\subsubsection{Shells}

This class of substructures is highly dependent on the projection effects. The middle panel of Fig. 9 (XZ-projection) demonstrates this result in particular. The incidence of shells is much higher in this projection than in the other two orientations. In Fig. 4 can be appreciated shells in only one orientation. The explanation is that shells are aligned with each other, either along one axis (prolate case) or a common equatorial plane (oblate case) of the central galaxy (Dupraz \& Combes 1986).

\section{Discussion}

Table 2 summarizes some of our findings and the characteristics of the three classes of fine structures. Colors are not considered in this paper given the difficulty to derive them in real observations, although they are provided in the simulations. With the deeper surface brightness limit, it is possible to detect shells in the most favorable orientation up until $4 \mathrm{Gyr}$, stellar streams when they are not destroyed by further minor mergers and until $3 \mathrm{Gyr}$; and tidal tails up until 2 Gyr. Tidal tails are considered the main tracer of major merger but they vanish more quickly. Streams are thinner and more frequent since they are produced
Table 2. Summary of results: properties of fine structures.

\begin{tabular}{lccc}
\hline \hline Property & Tidal tails & Shells & Streams \\
\hline $\begin{array}{l}\text { Formation } \\
\text { mechanism }\end{array}$ & $\begin{array}{c}\text {-1st and 3rd peak: } \\
\text { mass ratio }(1: 8)\end{array}$ & $\begin{array}{c}-1 \text { st peak: } \\
\text { ratio }(1: 8)\end{array}$ & $\begin{array}{c}-1 \text { st peak: } \\
\text { ratio }(1: 3)\end{array}$ \\
& $\begin{array}{c}\text { 2nd and 4th peak: } \\
(1: 3,1: 1.55)\end{array}$ & $\begin{array}{c}-2 \text { nd peak: } \\
\text { ratio }(1: 1.55)\end{array}$ & $\begin{array}{c}-2 \text { nd peak: } \\
\text { ratio }(1: 8)\end{array}$ \\
\hline Visibility & $0.7-1 \mathrm{Gyr}$ & $3-4 \mathrm{Gyr}$ & $1.5-3 \mathrm{Gyr}$ \\
\hline $\begin{array}{l}\text { Surface } \\
\text { brightness } \\
\text { sensitivity }\end{array}$ & No & Mild & High \\
\hline $\begin{array}{l}\text { Orientation } \\
\text { dependency }\end{array}$ & No & High & No \\
\hline Color $(B-V)$ & Blue: & Red: & Blue: \\
& -2.5 to -1.5 & -1.5 to 0 & -3 to -1 \\
\hline
\end{tabular}

in minor mergers, which have a much greater occurrence. Shells are highly dependent on sky orientation and they are the oldest vestiges of intermediate-mass mergers. In addition, their number increases over time, although their surface brightness decreases simultaneously.

It is interesting to compare these results with previous studies. Lotz et al. (2008) and Ji et al. (2014) identify different timescales for different merger stages of their simulated pair encounters. From their series of hydrodynamical simulations of equal-mass gas-rich disc galaxy mergers, Lotz et al. (2008) find characteristic perturbed and asymmetric morphologies during the merging time, but quite relaxed ones afterwards. Their observability timescales for the resulting fine structures are dependent on numerous parameters (viewing angle, orbit geometry, gas fraction, etc.) but are typically found in the same order as the coalescence timescale. The remains of the intense starburst triggered by the major merger is more easy to see than morphological perturbations. Their merger remnants appear disk-like and dusty, while exhibiting a massive bulge. Our simulation is of a different character as it follows a series of minor mergers 
and gas accretion in a cosmological context that is undergone by a central galaxy which is become an early-type quenched object. The observability timescales are therefore more realistic, taking into account successive mergers which are capable of destroying the vestiges of previous ones.

Ji et al. (2014) also follow a series of mergers, but their study is based on different mass ratios and use hydrodynamical simulations. They consider the survival timescales of the fine structures according to the surface brightness limit and galaxy environment. When isolated, the survival time is, on average, twice the coalescence time for a surface brightness limit of $25 \mathrm{mag} \mathrm{arcsec}-2$, i.e. $\sim 2.5 \mathrm{Gyr}$. This timescale can be twice as long $(\sim 5 \mathrm{Gyr})$ for a deeper surface brightness limit of $28 \mathrm{mag} \operatorname{arcsec}^{-2}$. Again, our simulation is more realistic since the central galaxy is the result of several satellite mergers during its formation and evolution, with different mass ratios ranging from $25: 1$ to $1.5: 1$, making the system more chaotic. For a minor merger of a small mass ratio, the final fine structure timescale can be quite long since the coalescence timescale itself is longer than for a major merger. The occurrence of minor mergers is traced more frequently by stellar streams than by tidal tails (which tend to characterize major mergers).

The advantages of our method, relative to the systematic study of individual mergers, is that we can study the survival time of fine structures based on a complex merging history. The results are then directly comparable to observations because, in addition, we use the same classification method sensitive to LSB features. To counterbalance these advantages, there are obvious limits to our method: we focus on only one merger history, and more computational efforts will be required to obtain a wider set of statistics. Also, it is difficult to follow individual substructures from one snapshot to the other: we only count the total number of features, and there is some degeneracy when one feature is destroyed and another one created, resulting in the same number. To reduce the degeneracy, more frequent snapshots should be documented, which would prove more demanding for the process of visual classification.

We demonstrate how fine structures in the outer parts and stellar halos of galaxies can help to trace back the merger history and the mass assembly of present day galaxies. A study of stellar halos of Milky Way-like galaxies in the nearby universe, compared with Illustris simulations, have shown that the morphology of galaxies are, indeed, closely linked to the significance of their halos (Elias et al. 2018). Galaxies which demonstrate a small stellar halo mass relative to their total mass are disk galaxies which are still forming stars, whereas those with a large fraction of their mass in their halos are quenched. Simulations by Karademir et al. (2019) show that minor mergers enrich the stellar halos of galaxies much more than their centers and that they are able to increase the size of galaxy disks, as has been observed (e.g., Newman et al. 2012).

In recent years, machine learning algorithms have been developed to identify fine structures in galaxy halos, both in observations and simulations (e.g. Walmsley et al. 2019; Hendel et al. 2019). Comparisons to visual classifications provide promising results and these methods will be inevitable for large surveys in the future.

Mantha et al. (2019) propose a tool for identifying tidal debris in CANDELS deep fields and in the VELA simulations as well, which helps to disentangle viewing effects and the subjectivity of visual inspections. Automatic tools like this will help to improve the characterization of the merger rate as a function of redshift.

\section{Conclusions}

We used a hydrodynamical simulation that has been resimulated within a cosmological context in order to analyze and interpret the morphologies and survival timescales of fine structures, as well as tracers of mergers and of the mass assembly of present day early-type galaxies. We took a census of three types of fine structures based on visual inspection: tidal tails, stellar streams, and shells. The observation of the number of fine structures detected around the central galaxy versus time allows us to reconstruct its merger history. We find three phases in the central galaxy evolution, the second one characterized as a very quiet phase. The census of fine structures are taken in correspondence with the merger events that have been identified. This allows us to establish that:

- Tidal tails result from major mergers events (1.5:1), stellar streams from minor mergers (10:1) and shells from major and intermediate-mass mergers (4:1).

- Tidal tails and shells have long survival times, 2 Gyr and $\sim 3$ Gyr, respectively, but streams remains visible across all phases of galaxy evolution.

- The detection of stellar streams are highly dependent on the surface brightness limit. We see between two and three times more streams with a surface brightness cut of $33 \mathrm{mag} \mathrm{arcsec}^{-2}$ than with $29 \mathrm{mag} \operatorname{arcsec}^{-2}$.

- The detection of shells depends considerably on the projection angle and orientation on the sky plane.

Our results are compatible with previous simulations of comparable scope, however, conducting the zoom-in resimulation in a cosmological context introduces more realistic conditions to estimate the survival timescales of fine structures around today's early-type galaxies.

As for the observer's point of view, the reconstruction of the merger history is obtained more optimally with shells, which have longer timescales. The number of shells can also indicate the time that has passed since the last merger. Streams have shorter timescales, so when no fine structure is observed, this means either no event has occurred since $2 \mathrm{Gyr}$, or it has been limited to a very minor merger with a large angular momentum, which produces more streams than shells.

Acknowledgements. We thank the anonymous referee for their constructive comments, and David Valls-Gabaud for the helpful discussion. B. Mancillas was supported in part by the CONACYT grant, CVU 420397.

\section{References}

Adams, S. M., Zaritsky, D., Sand, D. J., et al. 2012, AJ, 144, 128 Amorisco, N. C. 2015, MNRAS, 450, 575

Argudo-Fernandez, M., Shen, S., \& Sabater, J. 2016, A\&A, 592, A30

Arp, H. 1966, ApJS, 14, 1

Atkinson, A. M., Abraham, R. G., \& Ferguson, A. M. N. 2013, ApJ, 765, 28

Bell, E. F., Wolf, C., Meisenheimer, K., et al. 2004, ApJ, 608, 752

Bender, R., Burstein, D., \& Faber, S. M. 1992, ApJ, 399, 462

Birdsall, C. K., \& Fuss, D. 1969, J. Comput. Phys., 3, 494

Bruzual, G., \& Charlot, S. 2003, MNRAS, 344, 1000

Bournaud, F., \& Combes, F. 2002, A\&A, 392, 83

Bournaud, F., \& Combes, F. 2003, A\&A, 401, 817

Bournaud, F., Jog, C. J., \& Combes, F. 2005, A\&A, 437, 69

Bournaud, F., Jog, C. J., \& Combes, F. 2007, A\&A, 476, 1179

Bullock, J. S., \& Johnston, K. V. 2005, ApJ, 635, 931

Cappellari, M., Emsellem, E., Krajnović, D., et al. 2011, MNRAS, 413, 813

Conselice, C. J. 2003, ApJS, 147, 1

Conselice, C. J., Bershady, M. A., \& Jangren, A. 2000, ApJ, 529, 886

Cooper, A. P., Cole, S., Frenk, C. S., et al. 2010, MNRAS, 406, 744

Cooper, A. P., D'Souza, R., Kauffmann, G., et al. 2013, MNRAS, 434, 3348

Cooper, A. P., Gao, L., Guo, Q., et al. 2015, MNRAS, 451, 2703 
Cox, T. J., Jonsson, P., Primack, J. R., \& Somerville, R. S. 2006, MNRAS, 373, 1013

Crnojević, D., Ferguson, A. M. N., Irwin, M. J., et al. 2013, MNRAS, 432, 832 Di Matteo, P., Combes, F., Melchior, A.-L., \& Semelin, B. 2007, A\&A, 468, 61 Duc, P.-A. 2017, Form. Evol. Galaxy Outskirts, 321, 180

Duc, P.-A., Cuillandre, J.-C., Karabal, E., et al. 2015, MNRAS, 446, 120

Dupraz, C., \& Combes, F. 1986, A\&A, 166, 53

Eisenstein, D. J., \& Hut, P. 1998, ApJ, 498, 137

Elias, L. M., Sales, L. V., Creasey, P., et al. 2018, MNRAS, 479, 4004

Feldmann, R., Mayer, L., \& Carollo, C. M. 2008, ApJ, 684, 1062

Ferguson, A. M. N., Irwin, M. J., Ibata, R. A., Lewis, G. F., \& Tanvir, N. R. 2002, AJ, 124, 1452

Ferrarese, L., Côté, P., Cuillandre, J.-C., et al. 2012, ApJS, 200, 4

Fioc, M., \& Rocca-Volmerange, B. 1999, ArXiv e-prints [arXiv:astro-ph/9912179]

Guo, Q., White, S., Boylan-Kolchin, M., et al. 2011, MNRAS, 413, 101

Helmi, A., \& White, S. D. M. 1999, MNRAS, 307, 495

Hendel, D., \& Johnston, K. V. 2015, MNRAS, 454, 2472

Hendel, D., Johnston, K. V., Patra, R. K., \& Sen, B. 2019, MNRAS, 486, 3604

Hilz, M., Naab, T., Ostriker, J. P., et al. 2012, MNRAS, 425, 3119

Hood, C. E., Kannappan, S. J., Stark, D. V., et al. 2018, ApJ, 857, 144

Janowiecki, S., Mihos, J. C., Harding, P., et al. 2010, ApJ, 715, 972

Ji, I., Peirani, S., \& Yi, S. K. 2014, A\&A, 566, A97

Jedrzejewski, R. I. 1987, MNRAS, 226, 747

Johnston, K. V., Majewski, S. R., Siegel, M. H., Reid, I. N., \& Kunkel, W. E. 1999, AJ, 118, 1719

Johnston, K. V., Bullock, J. S., Sharma, S., et al. 2008, ApJ, 689, 936

Kado-Fong, E., Greene, J. E., Hendel, D., et al. 2018, ApJ, 866, 103

Karademir, G. S., Remus, R.-S., Burkert, A., et al. 2019, MNRAS, 487, 318

Kaviraj, S., Peirani, S., Khochfar, S., Silk, J., \& Kay, S. 2009, MNRAS, 394, 1713

Kennicutt, Jr., R. C. 1998, ApJ, 498, 541

Krajnović, D., Emsellem, E., Cappellari, M., et al. 2011, MNRAS, 414, 2923

Levinson, F. H., \& Roberts, Jr., W. W. 1981, ApJ, 245, 465

Lotz, J. M., Jonsson, P., Cox, T. J., \& Primack, J. R. 2008, MNRAS, 391, 1137

Majewski, S. R., Skrutskie, M. F., Weinberg, M. D., \& Ostheimer, J. C. 2003, ApJ, 599, 1082

Malin, D. F. 1977, AAS Photo Bull., 16, 10

Malin, D. F. 1978, Nature, 276, 591

Malin, D. F., \& Carter, D. 1983, ApJ, 274, 534
Mantha, K. B., McIntosh, D. H., Ciaschi, C. P., et al. 2019, MNRAS, 486, 2643

Martig, M., Bournaud, F., Teyssier, R., \& Dekel, A. 2009, ApJ, 707, 250

Martig, M., Bournaud, F., Croton, D. J., Dekel, A., \& Teyssier, R. 2012, ApJ, 756, 26

Martínez-Delgado, D., Pohlen, M., Gabany, R. J., et al. 2009, ApJ, 692, 955

Martínez-Delgado, D., Gabany, R. J., Crawford, K., et al. 2010, AJ, 140, 962

McConnachie, A. W., Irwin, M. J., Ibata, R. A., et al. 2009, Nature, 461, 66

Michel-Dansac, L., \& Wozniak, H. 2004, A\&A, 421, 863

Mihos, J. C., Harding, P., Feldmeier, J., \& Morrison, H. 2005, ApJ, 631, L41

Miskolczi, A., Bomans, D. J., \& Dettmar, R.-J. 2011, A\&A, 536, A66

Naab, T., Johansson, P. H., Ostriker, J. P., \& Efstathiou, G. 2007, ApJ, 658, 710

Newman, A. B., Ellis, R. S., Bundy, K., \& Treu, T. 2012, ApJ, 746, 162

Pawlik, M. M., Wild, V., \& Walcher, C. J. 2016, MNRAS, 456, 3032

Peirani, S., Crockett, R. M., Geen, S., et al. 2010, MNRAS, 405, 2327

Peng, C. Y., Ho, L. C., Impey, C. D., \& Rix, H.-W. 2002, AJ, 124, 266

Pop, A.-R., Pillepich, A., Amorisco, N. C., \& Hernquist, L. 2018, MNRAS, 480, 1715

Quinn, P. J. 1984, ApJ, 279, 596

Rodriguez-Gomez, V., Pillepich, A., Sales, L. V., et al. 2016, MNRAS, 458, 2371

Salpeter, E. E. 1955, ApJ, 121, 161

Schweizer, F. 1983, Int. Kinematics Dyn. Galaxies, 100, 319

Schweizer, F., \& Ford, Jr., W. K. 1985, New Aspects of Galaxy Photometry, 232, 145

Stewart, K. R., Bullock, J. S., Wechsler, R. H., Maller, A. H., \& Zentner, A. R. 2008, ApJ, 683, 597

Tal, T., \& van Dokkum, P. G. 2011, ApJ, 731, 89

Tal, T., van Dokkum, P. G., Nelan, J., \& Bezanson, R. 2009, AJ, 138, 1417

Teyssier, R. 2002, A\&A, 385, 337

Toomre, A. 1977, Evolution of Galaxies and Stellar Populations (New Haven: Yale University Observatory), 401

Toomre, A., \& Toomre, J. 1972, ApJ, 178, 623

Valls-Gabaud, D., \& MESSIER Collaboration 2017, Form. Evol. Galaxy Outskirts, 321, 199

van Dokkum, P. G. 2005, AJ, 130, 2647

van Dokkum, P. G., Abraham, R., \& Merritt, A. 2014, ApJ, 782, L24

Walmsley, M., Ferguson, A. M. N., Mann, R. G., et al. 2019, MNRAS, 483, 2968

Wen, Z. Z., \& Zheng, X. Z. 2016, ApJ, 832, 90

White, S. D. M., \& Rees, M. J. 1978, MNRAS, 183, 341

York, D. G., Adelman, J., Anderson, Jr., J. E., et al. 2000, AJ, 120, 1579 


\section{Appendix A: Snapshot evolution}

In the following three figures (Figs. A.1-A.3), we present the whole ensemble of snapshots of the zoom-in simulation of the three projections.

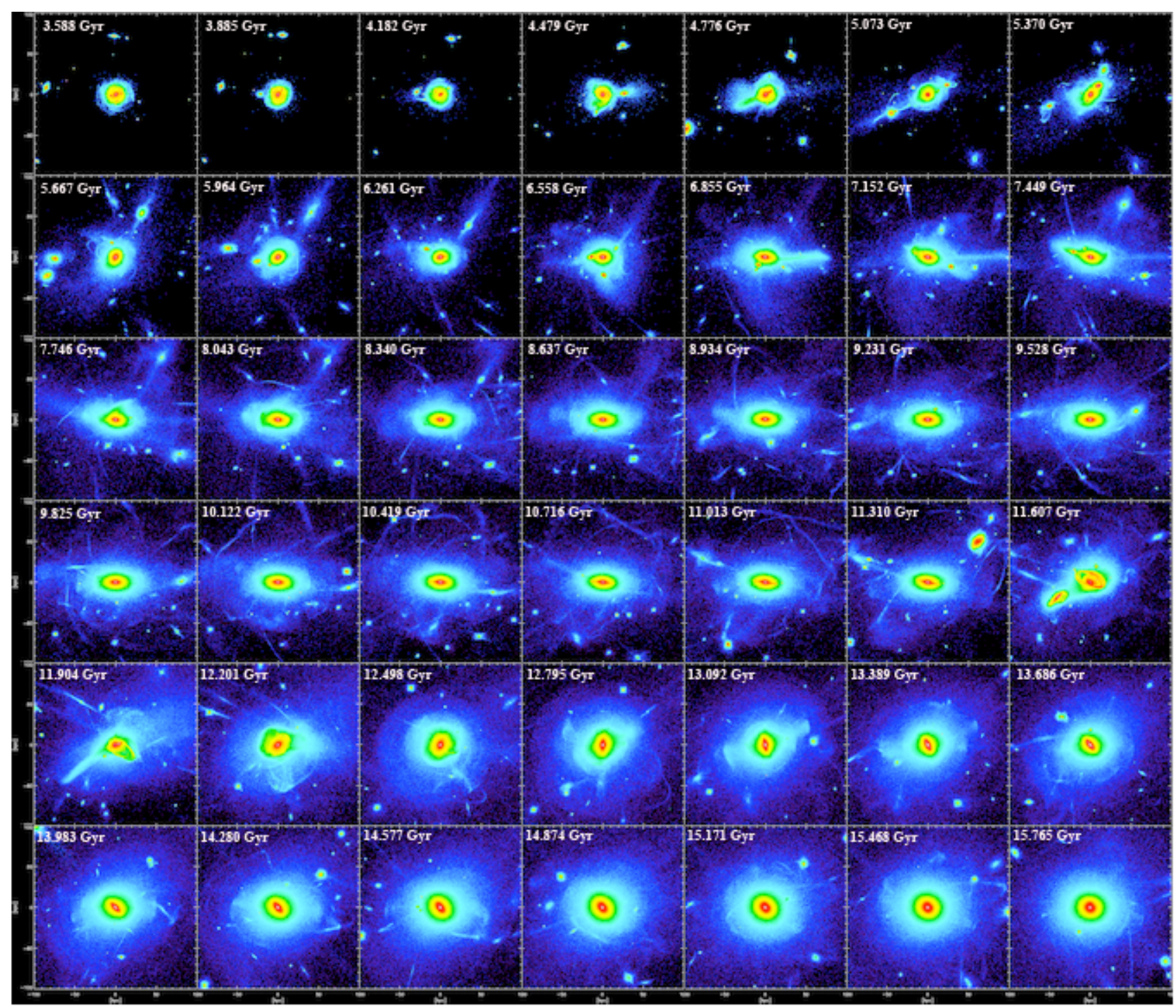

Fig. A.1. Snapshot evolution in XY-projection. 


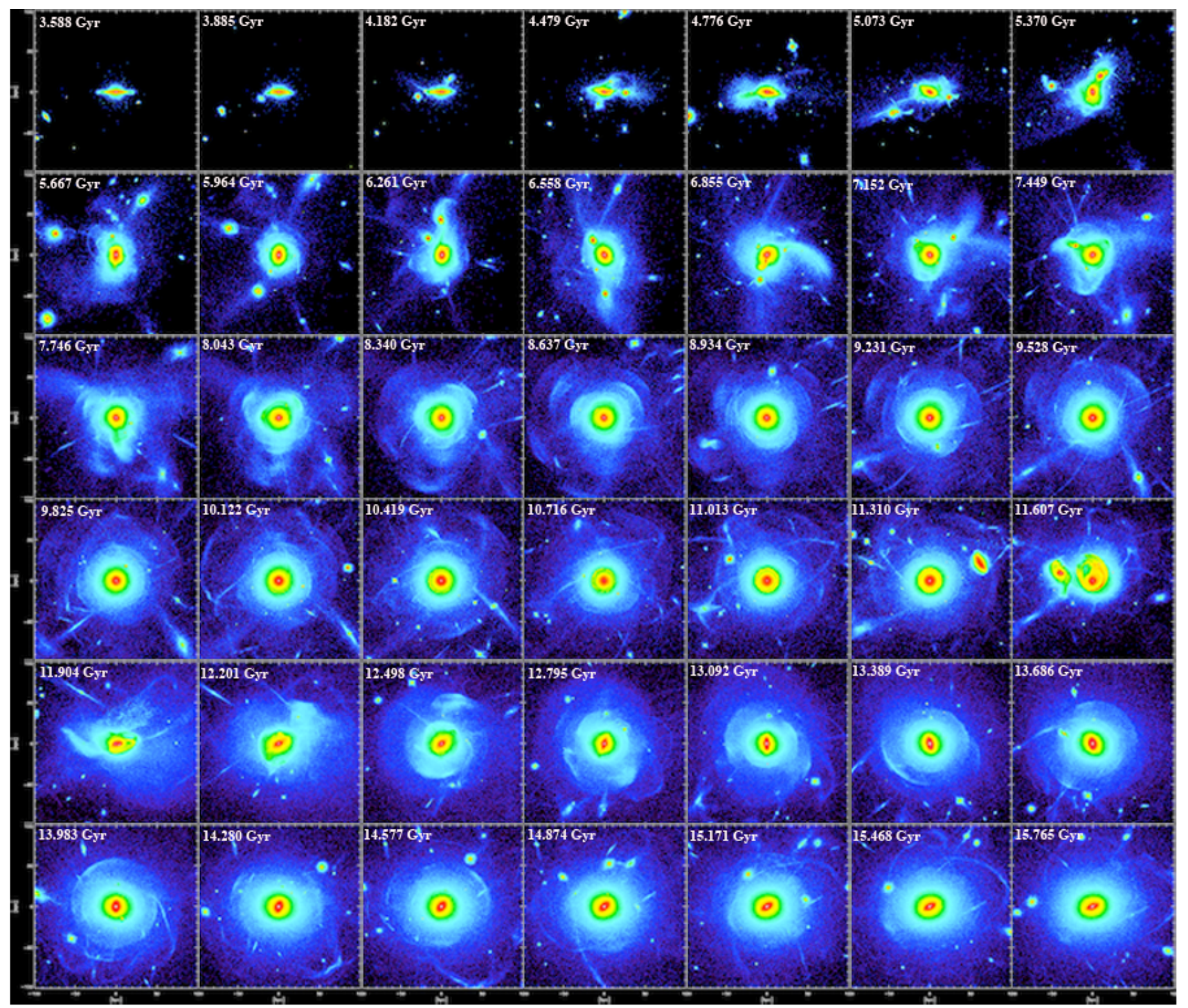

Fig. A.2. Snapshot evolution in YZ-projection. 
B. Mancillas et al.: Faint stellar substructures and merger history

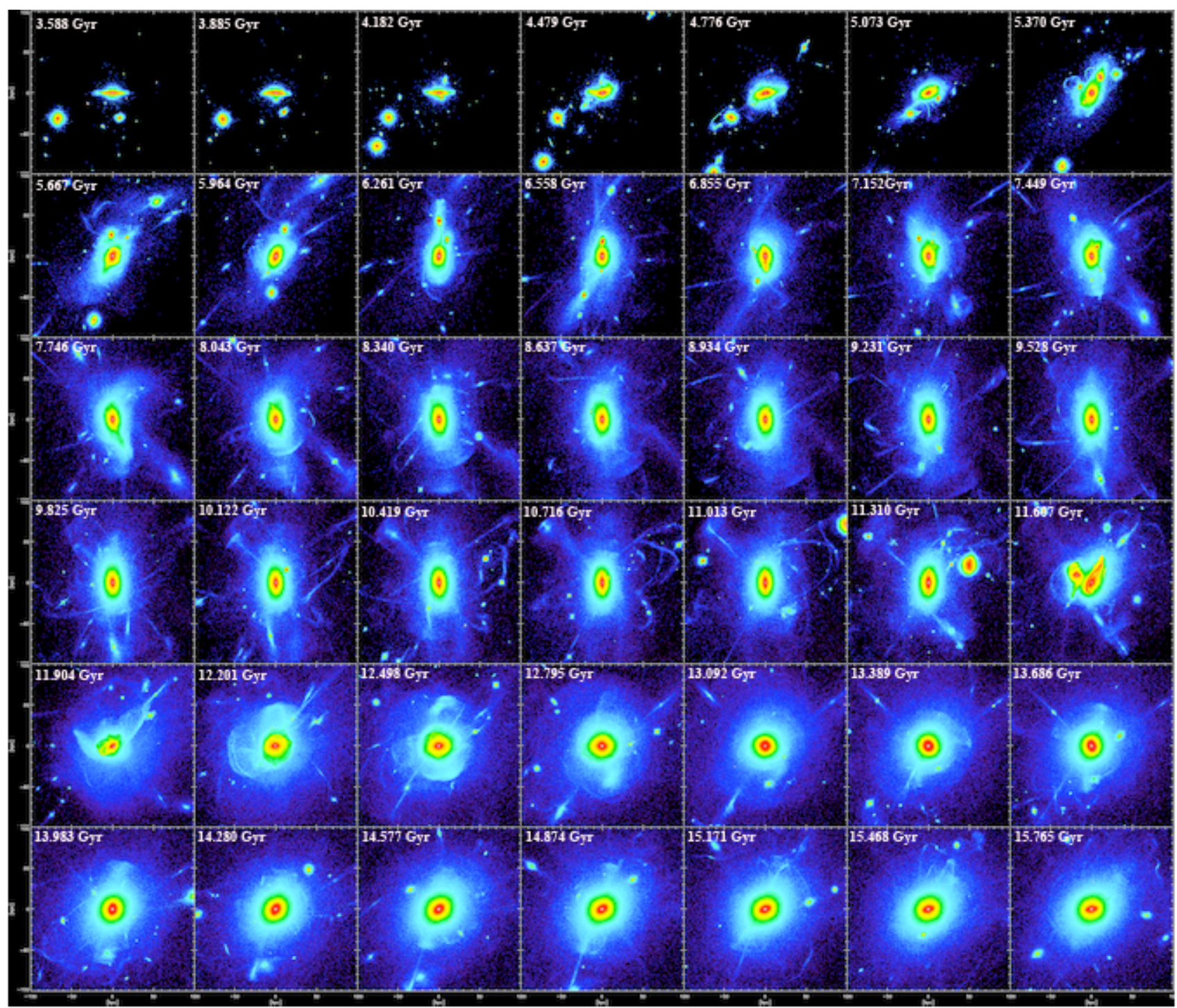

Fig. A.3. Snapshot evolution in the XZ-projection. 